\title{
Nonequilibrium electron rings for synchrotron radiation production
}

\author{
Hywel Owen* \\ The University of Manchester and Cockcroft Institute, Manchester M13 9PL, United Kingdom \\ Peter H. Williams \\ STFC Daresbury Laboratory and Cockcroft Institute, Daresbury Science and Innovation Campus, \\ Warrington WA4 4AD, United Kingdom
}

Scott Stevenson

The University of Oxford, Oxford OX1 2JD, United Kingdom

(Received 21 December 2012; published 9 April 2013; corrected 11 April 2013)

\begin{abstract}
Electron storage rings used for the production of synchrotron radiation (SR) have an output photon brightness that is limited by the equilibrium beam emittance. By using interleaved injection and ejection of bunches from a source with repetition rate greater than $1 \mathrm{kHz}$, we show that it is practicable to overcome this limit in rings of energy $\sim 1 \mathrm{GeV}$. Sufficiently short kicker pulse lengths enable effective currents of many milliamperes, which can deliver a significant flux of diffraction-limited soft x-ray photons. Thus, either existing SR facilities may be adapted for nonequilibrium operation, or the technique applied to construct SR rings smaller than their storage ring equivalent.
\end{abstract}

The storage ring-developed from the synchrotron $[1,2]$ —overcomes the relativistic limitation of fixed-target particle physics experiments by colliding counterrotating beams of particles [3,4]. The emitted synchrotron radiation (SR) [5] from cycling electron synchrotrons was already used parasitically from the 1970s for techniques such as x-ray crystallography [6-8]. Second- and third-generation facilities [9] use storage rings; third-generation rings provide magnet-free straight sections with zero (or small) dispersion [10,11], and may accommodate periodic magnetic insertion devices (IDs) that induce strong (and sometimes coherent) photon emission at selected wavelengths. The use of electron storage rings for SR blossomed in the 1980s and is now a mature field with around 60 facilities conducting a myriad of experiments [12,13]. Electron beam quality is characterized by the emittances $\epsilon_{x}$ and $\epsilon_{y}$, where, for a single dipole radius $\rho, \epsilon_{x}=$ $55 \hbar \gamma^{2}\left\langle H_{x}\right\rangle_{s} / 32 \sqrt{3} m_{e} c J_{x} \rho$ is determined by the energy (via $\gamma$ ) and by the magnetic lattice via $\left\langle H_{x}\right\rangle_{s}=\left\langle\beta_{x} \eta_{x}^{\prime 2}+\right.$ $\left.2 \alpha_{x} \eta_{x} \eta_{x}^{\prime}+\gamma_{x} \eta_{x}^{2}\right\rangle_{s}\left(J_{x}\right.$ is the horizontal damping partition number). Demand for better resolution pushes ring design to ever-smaller $\boldsymbol{\epsilon}_{x}$ using lattices that minimize $\left\langle H_{x}\right\rangle_{s}$ (and thereby the equilibrium between radiation damping and quantum excitation), for example, the theoretical minimum emittance (TME) lattice [14-17] in which only a small fraction of the circumference $C$ may be used for IDs. $\epsilon_{y}$ is

Published by the American Physical Society under the terms of the Creative Commons Attribution 3.0 License. Further distribution of this work must maintain attribution to the author(s) and the published article's title, journal citation, and DOI. coupled to $\epsilon_{x}$ mainly by ring magnet roll misalignments $[18,19]$ : originally $10 \%$ for the European Synchrotron Radiation Facility (Grenoble, France) [20,21], coupling values better than $0.1 \%$ are achieved today [22], smaller than the diffraction limit for many IDs. One may also increase $C$ to incorporate more dipoles to minimize $\left\langle\eta_{x}\right\rangle$ in them [23-25], conceptually simple but expensive and yielding only modest gains: it is therefore unlikely that rings much larger than the $\sim 1 \mathrm{~km}$ APS, ESRF, and SPRING-8 will be built (PETRA-III is a special case of a large preexisting ring for particle physics [26]).

Fourth-generation SR facilities overcome some limitations of storage rings, particularly, the linac-driven freeelectron laser (FEL) [27-29] first demonstrated at x-ray wavelengths at the Linac Coherent Light Source (Stanford, California) [30,31]. While FELs may provide enormous peak brightness, their average flux is rather modest in comparison with storage rings due to the limitations in either gun or linac macropulse repetition rate. Average flux may be increased by using superconducting cavities and highcurrent electron injectors: the energy-recovery linac (ERL) uses both to provide quasicontinuous bunch trains while alleviating power requirements in the cavities.Each electron's energy is recycled as the returned bunches are decelerated and then passed to new electrons [32,33]. Jefferson Lab has demonstrated $10 \mathrm{~kW}$ of FEL power at $100 \mathrm{MeV}$ electron energy in a $10 \mathrm{~mA}$ ERL [34,35].

Low-energy ERLs may circulate large currents without the strong intrabeam scattering (IBS) that would limit the emittance of a storage ring at the same energy [36,37]. At sufficiently large electron energies ERLs gain an emittance advantage over that possible from a storage ring. We recall 


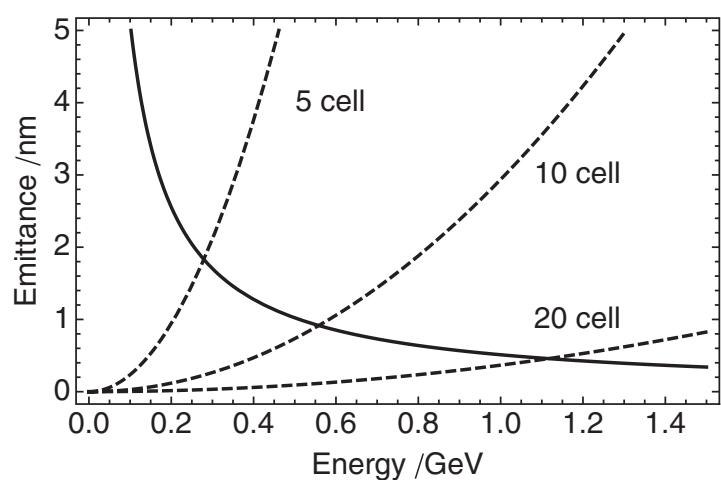

FIG. 1. Variation of circulating beam emittance in a storage ring versus energy, comparing an injector (with normalized emittance $\epsilon_{n}=10^{-6} \mathrm{~m}$ ) with equilibrium emittances for storage rings composed from TME double-bend achromats of 5, 10, and 20 cells (dashed lines).

that in the absence of dilution the emittance $\epsilon=\epsilon_{n} / \beta \gamma$ reduces adiabatically under acceleration. In Fig. 1 we compare the achievable emittances from a typical modern electron injector to TME lattices of differing size. This emittance advantage has led to several proposed facilities (e.g., Ref. [38]), including the conversion of existing storage rings $[39,40]$. However, since each electron contributes only once to the effective current, the charge passing through the accelerator is very high and beam loss control will be difficult. Several proposals assume tens of MWs of circulating beam power and losses kept to a few $\mathrm{W} / \mathrm{m}$ : for example, a $6 \mathrm{GeV}$ ERL circulating $100 \mathrm{~mA}$ through $1 \mathrm{~km}$ deposits $600 \mathrm{~kW}$ continuously if losses are kept to typical values $\sim 0.1 \%$, whereas the equivalent storage ring would deposit only $2000 \mathrm{~J}$ if a fill were completely lost. An ERL cavity injection scheme may be used to circulate each injected bunch four times instead of just once [41], but extending that concept is very challenging.

All present-day electron storage rings circulate bunches where classical SR damping and quantum excitation give an equilibrium emittance $\epsilon_{\mathrm{eq}(x, y)}$, the stored charge either accumulated over repeated bunch injection or transferred in one train from another storage ring. The injected emittances $\epsilon_{\mathrm{inj}(x, y)}$ are usually larger than $\epsilon_{\mathrm{eq}(x, y)}$, particularly in the vertical plane, although this need not be the case [42]. At energies above $\sim 1 \mathrm{GeV}$ the damping time $\tau_{x, y}=$ $3 m_{e}^{3} c^{5} C \rho /\left(2 \pi r_{e} J_{x, y} E^{3}\right)$ falls to a few milliseconds: linear collider damping rings use this to take a (relatively) large injector emittance and reduce it; bunches are left in just long enough to sufficiently damp before they are passed to a downstream linac.

We propose the opposite process, in which lowemittance bunches are repeatedly injected into a ring and grow larger: they are ejected and replaced with new ones well before equilibrium is obtained, so that the effective emittance is essentially $\epsilon_{\text {inj }}$. This process may be carried out in any of the radiofrequency (rf) buckets, limited by the
TABLE I. Principal properties of the MAX-II, MAX-III, and Super-ACO storage rings when operated in equilibrium.

\begin{tabular}{lccc}
\hline \hline & MAX-II & MAX-III & Super-ACO \\
\hline Maximum energy $(\mathrm{GeV})$ & 1.5 & 0.7 & 0.8 \\
Design current $(\mathrm{mA})$ & 200 & 250 & 400 \\
$C(\mathrm{~m})$ & 90 & 36 & 72 \\
$\rho(\mathrm{m})$ & 3.33 & 3.036 & 1.7 \\
$T_{\text {rev }}(\mathrm{ns})$ & 300 & 120 & 240 \\
$\epsilon_{\mathrm{eq},(x)}(\mathrm{nm})$ & 8.9 & 12.8 & 38.0 \\
$\tau_{x, y}(\mathrm{~ms})$ & 6.7 & 24 & 18 \\
$\sigma_{t}(1 \sigma, \mathrm{ps})$ & 53 & 89 & 90 \\
$\sigma_{E}(1 \sigma)\left(/ 10^{-4}\right)$ & 7.1 & 8.6 & 5.3 \\
$J_{x}$ & 1.0 & 2.4 & 1.0 \\
\hline \hline
\end{tabular}

injection rate. This concept has been proposed to improve Compton photon production [43] and for electron cooling [44], but not yet proposed for the production of SR. At large enough energies $\tau_{x, y}$ will be too short to allow repeated injection at a feasible rate. However, here we show that an energy range exists between a lower-energy emittance crossover point and the damping rate limit where there is an emittance advantage with reasonable average output flux.

We illustrate this nonequilibrium (NEQ) approach using the designs of the existing Swedish MAX-II [45,46] and MAX-III storage rings [47] and the French Super-ACO ring [48,49], which in normal operation have the properties given in Table I; the energy of each ring may be readily lowered to reduce $\epsilon_{\mathrm{eq}(x)}$ and increase $\tau_{x, y}$. We assume that full-energy injection of bunches with charge $q=1 \mathrm{nC}$ is possible at a frequency $f_{l}$ up to $10 \mathrm{kHz}$, from a suitable injector with $\epsilon_{n}=10^{-6} \mathrm{~m}$ : several FEL proposals indicate these parameters are accessible [50,51]. We consider the use of fast bunch-by-bunch injection and extraction as proposed for collider damping rings, for example, the $0.73 \mathrm{~m}$ DAFФNE and $1.4 \mathrm{~m}$ KEK kicker designs where short enough rise or fall times (12.4 and $6 \mathrm{~ns})$ and peak-topeak $(p-p)$ stability of $0.07 \%$ are possible with deflection angles at $1 \mathrm{GeV}$ of 2.6 and $3.0 \mathrm{mrad}$, respectively [52,53]; continuous repetition rates over $1 \mathrm{kHz}$ have been proposed for other fast kickers [51,54]. Over straight lengths of a few meters, the total kick angle from several kickers $(>5 \mathrm{mrad}$ for $E<1 \mathrm{GeV}$ ) is sufficient to translate the beam at least $10 \mathrm{~mm}$ from an injection septum, thereby providing ample Touschek and quantum lifetime for the circulating beam.

There is no significant radiation damping, since by definition electrons are resident for a time smaller than $\tau_{x, y}$. Emittance dilution due to injection steering errors will persist, and may be estimated as $\Delta \epsilon / \epsilon=\left[\Delta x^{2}+\right.$ $\left.\left(\beta_{0} \Delta x^{\prime}+\alpha_{0} \Delta x\right)^{2}\right] / 2 \beta_{0} \epsilon$. Dilution is small at energies below $1 \mathrm{GeV}$ for achievable $p-p$ stabilities and may be suppressed by reducing $\beta_{0}$ at the injection point; other dilution effects such as optical mismatch and steering fluctuations are expected to be small [55]. 
TABLE II. Nonequilibrium operation of MAX-II, MAX-III, and Super-ACO, assuming an injected bunch length equal to the natural bunch length at full energy. The beam current in all cases is $83 \mathrm{~mA}$, limited by the $12 \mathrm{~ns}$ bunch spacing.

\begin{tabular}{lcccccc}
\hline \hline & \multicolumn{2}{c}{ MAX-II } & \multicolumn{2}{c}{ MAX-III } & \multicolumn{2}{c}{ Super-ACO } \\
Energy $(\mathrm{GeV})$ & 0.7 & 1.0 & 0.5 & 0.7 & 0.5 & 0.8 \\
\hline$n_{b}$ & 25 & 25 & 10 & 10 & 20 & 20 \\
$\tau_{r}(\mathrm{~ms})$ for $f_{l}=10 \mathrm{kHz}$ & 2.5 & 2.5 & 1.0 & 1.0 & 2.0 & 2.0 \\
$\tau_{x, y}(\mathrm{~ms})$ & 66 & 23 & 13,32 & $4.8,11.5$ & 74 & 18 \\
$\tau_{\text {IBS } x, \text { init }}(\mathrm{ms})$ & 13 & 22 & 2.0 & 3.8 & 4.8 & 11.7 \\
$\epsilon_{\text {eq, } x}(\mathrm{~nm})$ & 2.73 & 3.95 & 6.5 & 12.8 & 15.0 & 38.0 \\
$\epsilon_{\text {inj, }, x}, \epsilon_{\text {inj, }, y}(\mathrm{~nm})$ & 0.730 & 0.511 & 1.28 & 1.02 & 1.02 & 0.639 \\
$\epsilon_{d, \mathrm{crit}}(\mathrm{nm})$ & 0.432 & 0.148 & 2.11 & 1.08 & 0.604 & 0.138 \\
$\Delta \epsilon / \epsilon(\mathrm{x}, \mathrm{y})$ from $p-p$ stability $(\%)$ & $16,2.7$ & $22,3.8$ & $1.0,0.7$ & $1.4,1.0$ & $5.0,5.8$ & $8.0,9.4$ \\
Dump power $(\mathrm{kW})$ for $f_{l}=10 \mathrm{kHz}$ & 7 & 10 & 5 & 7 & 5 & 8 \\
\hline \hline
\end{tabular}

The low energy and large bunch charges mean that IBS is important [56]. Growth rate estimates using ELEGANT [57] indicate initial characteristic times $\tau_{\text {IBS }} \lesssim 1 \mathrm{~ms}$ for injected bunch lengths $\sigma_{t} \sim 1 \mathrm{ps} ; \tau_{\mathrm{IBS}}$ varies linearly with $\sigma_{t}$, and so may be made greater than $\tau_{x, y}$ by increasing $\sigma_{t}$ from $1 \mathrm{ps}$ to the equilibrium stored lengths of either 50 or 90 ps. Large injected $\sigma_{t}$ may be achieved either with linac rf phasing or with bunch shear from a magnetic chicane. For large $\sigma_{t}$ the variation in deflection angle over the kicker pulse is still small, and even at $\sigma_{t} \sim 90 \mathrm{ps}$ the dilution effect on the circulating emittance is less than $1 \%$. Interleaved injection or extraction is therefore feasible, but the alternative scheme of repeatedly injecting bunch trains is not, since the much longer required kicker pulse will vary significantly in amplitude over the train. We note that other collective effects such as resistive wall instabilities, microbunching, and so on will either be small or controllable with feedback [58].

The number of bunches $n_{b}$ that may be circulated in NEQ mode is limited by the kicker rise or fall time rather than by $f_{l}$, as it is in damping rings. For $q=1 \mathrm{nC}$ the total current that may be circulated is simply $I=q / \tau_{b}$, where $\tau_{b}$ is the possible bunch spacing, i.e., $I=83 \mathrm{~mA}$ for $12 \mathrm{~ns}$ spacing (6 ns kicker rise and fall time), with $n_{b}=T_{\text {rev }} / \tau_{b}$; there is an insignificant reduction in effective $I$ due to the time taken to replace each bunch (which is smaller than $\left.T_{\text {rev }}\right)$. The residence time of each bunch in the ring is determined by the repetition rate $f_{l}$ of the injector as $\tau_{r}=n_{b} / f_{l}$. With a $1 \mathrm{kHz}$ injector rate the residence times are long enough that IBS would determine the emittance, but at $f_{l}=10 \mathrm{kHz} \tau_{r}$ is small enough that no significant emittance growth occurs. Table II gives example operating energies for each of the considered rings and shows that a significant emittance advantage may be obtained using NEQ operation.

IDs will deliver photons comparable to the critical wavelength $\lambda_{c}=4 \pi \rho / 3 \gamma^{3}$ of the main ring dipoles: we may therefore use the diffraction-limited emittance at $\lambda_{c}$, given as $\epsilon_{d \text {,crit }}=\rho / 3 \gamma^{3}$, as an estimate of the useful emittance from NEQ operation; this is compared to the equilibrium and NEQ emittances in Fig. 2. Equating $\epsilon_{d \text {,crit }}$ with $\epsilon_{\text {inj }}$, we obtain the electron energy at which NEQ operation gives an advantage, $E \simeq m_{e} c^{2} \sqrt{\rho / 3 \epsilon_{n}}$, around 0.4-0.7 GeV for typical dipole radii. The upper limit on $E$ is set by ensuring $\tau_{r} \ll \tau_{x}$, in other words, that $f_{l} \gg$ $2 \pi r_{e} J E^{3} / 3 m_{e}^{3} c^{6} \rho \tau_{b}$; this limit is about $1.5-2.5 \mathrm{GeV}$ depending on $\rho$, and is similar to the energy limit from kicker emittance dilution. The beam power deposited at the dump is simply that from the injector, and is readily manageable even at $f_{l}=10 \mathrm{kHz}$ [38,51,59-61]: the beam power is therefore limited by the kicker rise or fall time. A significant beam power saving is obtained over an equivalent ERL, with no emittance penalty. Compared to an equilibrium storage ring, the horizontal emittance is reduced significantly with NEQ operation and may be brought close to $\epsilon_{d \text {,crit }}$; the equilibrium vertical emittance is typically smaller than $\epsilon_{d \text {,crit }}$, so the increase from NEQ operation incurs no penalty for most IDs. The range of output photon energies over which NEQ operation gives an

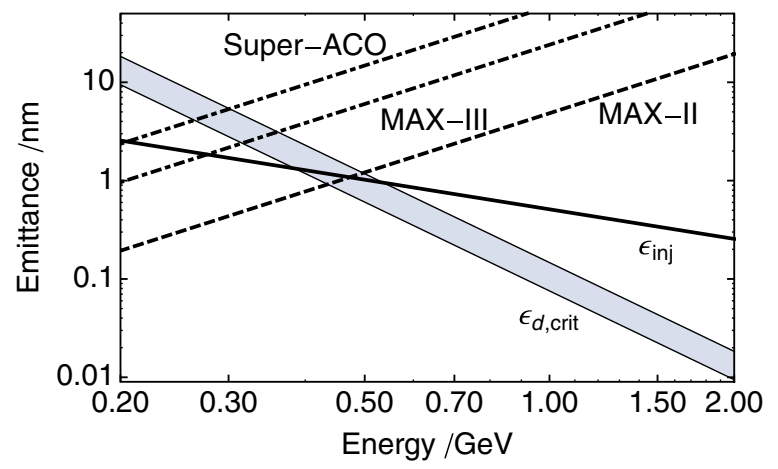

FIG. 2 (color online). Variation of emittance with energy, comparing an injector (with normalized emittance $\epsilon_{n}=$ $10^{-6} \mathrm{~m}$ ) with the equilibrium emittance of the MAX-II, MAX-III, and Super-ACO storage rings. Also shown is the range of the diffraction-limited emittance $\epsilon_{d \text {, crit }}$ for bending radii $\rho$ from 1.7 to $3.33 \mathrm{~m}$. 
emittance advantage is from about $\sim 0.1 \mathrm{keV}$ to $\sim 2 \mathrm{keV}$. We suggest that NEQ rings of simple optical design and small $\rho$ may be an inexpensive way to add spontaneous photon beam lines to a linac that drives a soft $\mathrm{x}$-ray or higher-energy FEL. A small circumference lattice can give NEQ emittances similar to much larger rings operated in equilibrium while still delivering simultaneous photons with significant flux to numerous IDs and their associated experiments.

This work was supported in part by the Science and Technology Facilities Council. We would like to thank Jim Clarke and James Jones of STFC Daresbury Laboratory and Sara Thorin of MAX-Lab for technical information and useful discussions.

*hywel.owen@manchester.ac.uk

[1] E. McMillan, Phys. Rev. 68, 143 (1945).

[2] F. K. Goward and D. E. Barnes, Nature (London) 158, 413 (1946).

[3] G. O’Neill, Phys. Rev. 102, 1418 (1956).

[4] G. O’Neill, Science 141, 679 (1963).

[5] J. Schwinger, Phys. Rev. 75, 1912 (1949).

[6] E. Rowe and F. Mills, Part. Accel. 4, 211 (1973).

[7] J. Worgan, Nucl. Instrum. Methods Phys. Res. 195, 49 (1982).

[8] V. Suller and D. Thompson, Nucl. Instrum. Methods 152, 1 (1978).

[9] D. Thompson, Nucl. Instrum. Methods 177, 43 (1980).

[10] D. Thompson, Nucl. Instrum. Methods Phys. Res. 201, 1 (1982).

[11] D. Thompson, Nucl. Instrum. Methods Phys. Res. 208, 1 (1983).

[12] J. L. Laclare, Nucl. Instrum. Methods Phys. Res., Sect. A 467-468, 1 (2001).

[13] D. Bilderback, P. Elleaume, and E. Weckert, J. Phys. B 38, S773 (2005).

[14] S. Lee and L. Teng, in Proceedings of the 14th Particle Accelerator Conference (IEEE, San Francisco, 1991), p. 2679.

[15] T. Lee and J. Choi, Nucl. Instrum. Methods Phys. Res., Sect. A 515, 410 (2003).

[16] T. Lee and J. Choi, Nucl. Instrum. Methods Phys. Res., Sect. A 534, 371 (2004).

[17] C.-X. Wang, Phys. Rev. ST Accel. Beams 12, 061001 (2009).

[18] A. Loulergue and J. Payet, in Proceedings of the 6th European Particle Accelerator Conference (IOP, Stockholm, 1998), p. 918.

[19] J. A. Clarke et al., in Proceedings of the 19th Particle Accelerator Conference (IEEE, Chicago, 2001), p. 2611.

[20] K. Witte, Synchrotron Radiation News 3, 6 (1990).

[21] ESRF Foundation Phase Report (Red Book), ESRF Technical Report, 1987.

[22] R. Bartolini, I. P. S. Martin, J. H. Rowland, P. Kuske, and F. Schmidt, Phys. Rev. ST Accel. Beams 11, 104002 (2008).

[23] A. Ropert et al., in Proceedings of the 7th European Particle Accelerator Conference, Vienna, 2000, p. 83.
[24] P. Elleaume and A. Ropert, Nucl. Instrum. Methods Phys. Res., Sect. A 500, 18 (2003).

[25] K. Tsumaki and N. Kumagai, Nucl. Instrum. Methods Phys. Res., Sect. A 565, 394 (2006).

[26] K. Balewski, W. Brefeld, W. Decking, Y. Li, G. Sahoo, and R. Wanzenberg, in Proceedings of the 9th European Particle Accelerator Conference (European Physical Society Accelerator Group, Lucerne, 2004), p. 2302.

[27] P. H. Fuoss, Nucl. Instrum. Methods Phys. Res., Sect. A 264, 497 (1988).

[28] H. Winick et al., Nucl. Instrum. Methods Phys. Res., Sect. A 347, 199 (1994).

[29] J. Arthur, G. Materlik, R. Tatchyn, and H. Winick, Rev. Sci. Instrum. 66, 1987 (1995).

[30] P. Emma et al., in Proceedings of the 23rd Particle Accelerator Conference, Vancouver, 2009, p. 3115.

[31] D. Ratner et al., in Proceedings of the Free Electron Laser Conference (FEL2009), Liverpool, 2009, 221.

[32] G. Neil and L. Merminga, Rev. Mod. Phys. 74, 685 (2002).

[33] W. R. Flavell et al., Proc. SPIE Int. Soc. Opt. Eng. 5917, 59170C (2005).

[34] G. Neil et al., Phys. Rev. Lett. 84, 662 (2000).

[35] G. Neil et al., Nucl. Instrum. Methods Phys. Res., Sect. A 557, 9 (2006).

[36] G. Hoffstaetter, V. Litvinenko, and H. L. Owen, Nucl. Instrum. Methods Phys. Res., Sect. A 557, 345 (2006).

[37] S. Smith et al., Nucl. Instrum. Methods Phys. Res., Sect. A 557, 145 (2006).

[38] E. A. Seddon et al., 4GLS Conceptual Design Report, CCLRC Technical Report, 2006.

[39] G. Hoffstaetter et al., Cornell Energy Recovery Linac Project Definition Design Report, Cornell University Technical Report, 2012.

[40] M. Borland, G. Decker, A. Nassiri, Y.-e. Sun, and M. White, Nucl. Instrum. Methods Phys. Res., Sect. A 582, 54 (2007).

[41] T. Nakamura, Phys. Rev. ST Accel. Beams 11, 032803 (2008).

[42] M. Boege et al., in Proceedings of the 6th European Particle Accelerator Conference (IOP, Stockholm, 1998), p. 623.

[43] P. Yu, Y. Wang, and W. Huang, Phys. Rev. ST Accel. Beams 12, 061301 (2009).

[44] Y. Derbenev et al., in Proceedings of the Workshop on Beam Cooling and Related Topics (COOL'07) (Gesellschaft für Schwerionenforschung $\mathrm{GmbH}$, Bad Kreuznach, 2007), p. 187.

[45] M. Sjostrom, H. Tarawneh, E. Wallen, and M. Eriksson, Nucl. Instrum. Methods Phys. Res., Sect. A 577, 425 (2007).

[46] G. LeBlanc and L. J. Lindgren, in Proceedings of the 5th European Particle Accelerator Conference (IOP, Sitges, 1996).

[47] M. Sjostrom, E. Wallen, M. Eriksson, and L. J. Lindgren, Nucl. Instrum. Methods Phys. Res., Sect. A 601, 229 (2009).

[48] M. E. Couprie, M. Velghe, R. Prazeres, D. Jaroszynski, and M. Billardon, Phys. Rev. A 44, 1301 (1991).

[49] J. L. Zyngier et al., IEEE Trans. Nucl. Sci. 32, 3371 (1985). 
[50] F. Sannibale et al., Phys. Rev. ST Accel. Beams 15, 103501 (2012).

[51] G. Aeppli et al., New Light Source (NLS) Project: Conceptual Design Report, Science and Technology Facilities Council Technical Report, 2009.

[52] D. Alesini, F. Marcellini, S. Guiducci, and P. Raimondi, Fast injection kickers for DAFNE and ILC damping rings, LNF-INFN EUROTeV-Report-No. 2006-025, 2006.

[53] T. Naito, H. Hayano, M. Kuriki, N. Terunuma, and J. Urakawa, Nucl. Instrum. Methods Phys. Res., Sect. A 571, 599 (2007).

[54] M. Placidi et al., in Proceedings of the 3rd International Particle Accelerator Conference (IEEE, New Orleans, 2012), p. 2053.

[55] W. Fischer, W. Mackay, S. Peggs, and J. Wei, Part. Accel. 58, 181 (1997).
[56] K. Kubo, S. K. Mtingwa, and A. Wolski, Phys. Rev. ST Accel. Beams 8, 081001 (2005).

[57] M. Borland, Advanced Photon Source Technical Report No. LS-287.

[58] K. M. Hock and A. Wolski, Phys. Rev. ST Accel. Beams 12, 091001 (2009).

[59] M. Wiseman et al., in Proceedings of the 17th Particle Accelerator Conference, Vancouver, 1997 (IEEE, New York, 1997), p. 3761.

[60] M. Maslov, M. Schmitz, and V. Sychev, Layout considerations on the $25 \mathrm{GeV} / 300 \mathrm{~kW}$ Beam Dump of the XFEL Project, DESY Technical Report No. TESLA-FEL 2006005, 2005.

[61] J. L. Fernandez-Hernando and D. Angal-Kalinin, in Proceedings of the 1st International Particle Accelerator Conference (IPAC, Kyoto, 2010), p. 1805. 